\title{
Anethole dithiolethione improves liver fatty acid metabolism in hamster fed high-fat
}

\section{diets}

Chengcheng Zhao ${ }^{1,2}$, Nannan $\mathrm{Yu}^{1,2}$, Wenqun $\mathrm{Li}^{1,2}$, Hualin Cai ${ }^{1,2}$, Mouze Liu ${ }^{1,2}$, Yanjie $\mathrm{Hu}^{3}$, Yiping Liu ${ }^{1,2, \#}$, Mimi Tang ${ }^{4,5, \#}$

${ }^{1}$ Department of Pharmacy, the Second Xiangya Hospital, Central South University,

Changsha, Hunan 410011, PR China

${ }^{2}$ Institute of Clinical Pharmacy, Central South University, Changsha, Hunan 410011, PR

China

${ }^{3}$ Department of Stomatology, Suiyang County People's Hospital, Zunyi, Guizhou, 563300,

PR China

${ }^{4}$ Department of Pharmacy, Xiangya Hospital, Central South University, Changsha, Hunan 410008, PR China

${ }^{5}$ Institute for Rational and Safe Medication Practices, National Clinical Research Center for

Geriatric Disorders, Xiangya Hospital, Central South University, Changsha, Hunan, 410008,

PR China

Running title: ADT reduces excessive accumulation of toxic FAs in the liver

Correspondence: Yiping Liu (yipingliu1973cn@csu.edu.cn, phone + 0731-85294046 ) and

Mimi Tang (tangmimi1989@163.com, fax +0731-84327453 )

\section{Abbreviations:}

ADT: anethole dithiolethione, 5-(4-methoxyphenyl)-3H-1,2-dithiole-3-thione

ACC1: acetyl-CoA carboxylase1

CPT1 $\alpha$ : carnitine palmitoyltransferase $1 \alpha$ 
EFAs: essential fatty acids

FAs: fatty acids

FAS: fatty acid synthase

FADS1: delta-5 desaturases

FADS2: delta-6 desaturases

FAT/CD36: fatty acid transposase

FATP: fatty acid transport protein

$\mathrm{H}_{2} \mathrm{~S}$ : hydrogen sulfide

HFD: high-fat diet

L-FABP: liver fatty acid binding protein

Mitofusin1: mitochondrial fusion protein 1

MUFAs: monounsaturated fatty acids

NAFLD: non-alcoholic fatty liver disease

PUFAs: polyunsaturated fatty acids

SFAs: saturated fatty acids

SCD1: stearoyl-CoA desaturase1 


\section{Abstract}

"Lipotoxicity" induced by excessive accumulation of free fatty acids (FFAs) in the liver, especially saturated FAs and their toxic metabolites, is closely related to metabolic diseases such as nonalcoholic fatty liver disease (NAFLD). Hydrogen sulfide $\left(\mathrm{H}_{2} \mathrm{~S}\right)$, a novel gaseous signaling molecule, has been reported to have lipid-lowering effects, but its effect on FAs metabolism remains unclear. The purpose of this study was to investigate the effect and mechanisms of anethole dithiolethione (ADT, a sustained-release $\mathrm{H}_{2} \mathrm{~S}$ donor) on hepatic FAs metabolism. ADT was administered daily for 4 weeks in male Syrian golden hamsters fed a high fat diet (HFD), and FAs profiles of liver tissues were analyzed using GC-MS. The results showed that in HFD-fed hamsters, ADT treatment significantly reduced the accumulation of saturated and monounsaturated fatty acids (C16:0, C18:0, C16:1, and C18:1n9), while increased the content of n- 6 and n-3 series polyunsaturated fatty acids (C20:3n6, C20:4n6, and C22:6n3). Mechanistically, ADT obviously inhibited the overexpression of ACC1, FAS and SCD1, and up-regulated the levels of FATPs, L-FABP, CPT1 $\alpha$, FADS1 and FADS2. Notably, ADT evidently induced Mitofusin 1 to facilitate mitochondrial fusion and optimize $\beta$ oxidation. These findings suggest that ADT plays a beneficial role by regulating the synthesis, desaturation, $\beta$-oxidation, uptake, binding/isolation, and transport of FAs. In conclusion, ADT is effective in improving liver FAs metabolic disorders and liver injuries caused by HFD.

Keywords: lipotoxicity; mitochondrial; $\beta$-oxidation; fatty acid/bing protein; fatty acid/synthesis; fatty acid/desaturases; palmitic acid; oleic acid; arachidonic acid; docosahexaenoic acid; 


\section{Introduction}

Fatty acids (FAs) are indispensable sources of energy in cells, and also important bioactive mediators involved in many homeostasis processes, including metabolism and regulating inflammatory immune responses $(1,2)$. However, abnormal fatty acid metabolism (synthesis, desaturation, oxidation, absorption, transport) can lead to diseases such as hyperlipidemia, non-alcoholic fatty liver disease(NAFLD), diabetes, and atherosclerosis(3-6). The liver is the main metabolic organ and plays a vital role in maintaining the balance of fatty acid levels in the body. When the level of free FAs(mainly saturated palmitate) in the liver rises beyond its mitochondrial oxidation, heterotopic lipid deposition is induced, which is called "lipotoxicity"(5). There is growing evidence that lipotoxicity is mainly caused by longchain saturated fatty acids (SFAs), such as palmitic acid (PA, C16: 0) and stearic acid (SA, C18: 0), while monounsaturated fatty acids (MUFAs) are generally less toxic and Polyunsaturated fatty acids (PUFAs) may even be protective(7-9). Three major mechanisms have been reported in palmitate and stearic acid-mediated lipotoxicity: (i) increased synthesis of harmful complex lipids such as diacylglycerol (DAG) and ceramide; (ii) impaired endoplasmic reticulum and mitochondrial function; (iii) membrane receptor-mediated inflammation, such as toll-like receptor 4 (TLR4)(5, 10-14).

Palmitic acid (C16: 0), stearic acid (C18: 0), and palmitoleic acid (C16: 1), oleic acid (C18: 1), the most common SFAs and MUFAs in the human body, are available in the diet or synthesized endogenously by the liver from carbohydrates, amino acids, and other fatty acids. The key enzymes in this process are acetyl-CoA carboxylase1 (ACC1) and FA synthase (FAS), which add seven malonyl CoAs to acetyl CoA, generating palmitic acid. Palmitic acid 
can be further metabolized to palmitoleic acid, stearic acid, and oleic acid, through delta-9 desaturase (stearoyl-CoA desaturase1, SCD1) and elongase. Compared with SFAs and MUFAs, animals and humans are unable to synthesize linoleic acid (LA, C18:2n6) and $\alpha$ linolenic acid (ALA, C18:3n3) from precursors oleic acid(C18:1n9) due to the lack of delta12 and delta-15 desaturases, so they must be obtained from diets. LA and ALA use the same enzyme systems (such as delta-5 and delta-6 desaturases (FADS1, FADS2), elongase) to produce PUFAs, including n-6 (arachidonic acid, AA, C20:4n6) and n-3 PUFAs (eicosapentaenoic acid (EPA, C20:5n3) and docosahexaenoic acid (DHA, C22:6n3)). N-6 and n-3 PUFAs are precursors of biologically active lipid mediator signaling molecules, including eicosanoids, which play an important role in regulating pro-inflammatory and / or antiinflammatory / resolution processes (Figure 1).

Mitochondrial $\beta$-oxidation is the most important metabolic pathway of fatty acids and is mainly regulated by rate-limiting enzymes such as carnitine palmitoyl-transferase $1 \alpha$ (CPT $1 \alpha)$, which serves as a gatekeeper for fatty acids to enter mitochondria. In addition, only fused mitochondria can ensure that FAs are homogeneously distributed throughout the mitochondria, maximizing the use of FAs for $\beta$-oxidative reactions(15). However, in the mitochondrial fusion protein 1 knockout (Mitofusin1 KO) cells, mitochondria did not form a network and were fragmented, reducing the $\beta$-oxidation rate and leading to lipid accumulation in lipid droplets $(15,16)$.

Hydrogen sulfide $\left(\mathrm{H}_{2} \mathrm{~S}\right)$, a well-known novel gaseous signaling molecule, is increasingly recognized as a crucial regulator of cardiovascular diseases. Studies have shown that $\mathrm{H}_{2} \mathrm{~S}$ exerts significant protective effects on metabolic diseases (NAFLD, diabetes and 
atherosclerosis) through multiple properties, such as anti-inflammatory, antioxidant, inhibiting foam cell formation, improving endothelial function, and increasing insulin receptor sensitivity(17-20). $\mathrm{H}_{2} \mathrm{~S}$ has been reported to have a lipid-lowering effect by activating liver autophagy(21), but its effect on fatty acid metabolism remains unclear. NaHS, a so-called immediate-release $\mathrm{H}_{2} \mathrm{~S}$ donor, releases excessive $\mathrm{H}_{2} \mathrm{~S}$ instantaneously and therefore does not mimic the production of endogenous $\mathrm{H}_{2} \mathrm{~S}(22)$. Anethole dithiolethione (ADT; 5-(4methoxyphenyl)-3H-1,2-dithiole-3-thione), clinically used as a hepatoprotective and choleretic drug, is also a prodrug of $\mathrm{H}_{2} \mathrm{~S}$ and releases $\mathrm{H}_{2} \mathrm{~S}$ in a controlled manner within a few hours in the body $(23,24)$. This article investigates how ADT affects hepatic fatty acid metabolism and explores its possible mechanisms. 


\section{Materials and Methods}

\subsection{Drug}

ADT(Lot.N1017A) was purchased from Dalian Meilun Biotechnology Co., LTD., and its clinical dosage is $75 \mathrm{mg}$ per day. The dosage of ADT in Syrian golden hamsters was calculated by human equivalent dose (HED) based on body surface area(25), with a conversion dose of $9.25 \mathrm{mg} / \mathrm{kg}$. Therefore, the dose gradient of ADT in this study was set as: $5 \mathrm{mg} / \mathrm{kg}, 10 \mathrm{mg} / \mathrm{kg}$, $20 \mathrm{mg} / \mathrm{kg}$ and $40 \mathrm{mg} / \mathrm{kg}$. ADT is insoluble in water, suspended in $0.5 \%$ sodium carboxymethylcellulose (CMC-Na) and $0.5 \%$ soybean lecithin, administered daily by gavage.

\subsection{Animals and Diets}

This study was reviewed and approved by the Laboratory Animal Care and Welfare Committee of Central South University(Approval No. 2018sydw0215). Fifty-four male Syrian golden hamsters $(80-100 \mathrm{~g})$ were purchased from Beijing Vital River Laboratory Animal Technology Co., Ltd. (Qualified Certificate No. SCXK Jing 2016-0011). Hamsters were housed in IVC cages and free access to diet and water, which subjected to a 12-hour light/dark cycle with a relative humidity of $50 \% \pm 10 \%$ and a temperature of $22^{\circ} \mathrm{C}-25^{\circ} \mathrm{C}$.

Trophic Animal Feed High-Tech Co. Ltd provided high-fat diets and control diets, both of which are formulated based on purified ingredients. The control diet was designed to meet all of the hamster's nutritional requirements. The cholesterol and lard in the high-fat diet were 2/1000 g and 117/1000 g, respectively.

\subsection{Experimental design}

After adaptation for one week, 54 hamsters were randomly divided into six groups, with 9 animals in each group. (1) control group: control diet with vehicle $(0.5 \% \mathrm{CMC}-\mathrm{Na}$ and 
0.5\% soybean lecithin) treatment; (2) HFD group: high-fat diet with vehicle (0.5\% CMC-Na and $0.5 \%$ soybean lecithin) treatment; (3-6) HFD+ADT group: high-fat diet with different doses of ADT. ADT and/or vehicle were administered to the hamster by gavage once a day for 4 weeks. After 4 weeks, all hamsters fasted overnight and were euthanatized at the end of the experiment. Blood samples were collected from the heart and kept at room temperature for $1 \mathrm{~h}$ before centrifugation at $3500 \mathrm{rpm}$ for $10 \mathrm{~min}$. The serum was separated and stored at $-80^{\circ} \mathrm{C}$ until analysis. The left lobe of liver was fixed in $4 \%$ paraformaldehyde solution, and the rest was stored at $-80^{\circ} \mathrm{C}$ after quick-freezing by liquid nitrogen.

\subsection{Biochemical and Histological Analysis}

Serum glutamate alanine aminotransferase (ALT), aspartate aminotransferase (AST) and total bile acid (TBA) were measured by automatic biochemical analyzer(Hitachi 7600-210) in the laboratory of Second Xiangya Hospital. The fixed liver tissue was embedded in paraffin blocks, and 10- $\mu \mathrm{m}$-thick slices were cut and stained with hematoxylin and eosin (HE) to observe histopathological changes.

\subsection{Sample pretreatment and FFAs determination}

FFAs in liver tissue were extracted and measured by GC-MS (Agilent 7890 A / 5975 C) according to the method described by Tang et al(26). In short, $750 \mu 1$ of dichloromethane and methanol $\left(\mathrm{CH}_{2} \mathrm{Cl}_{2}: \mathrm{CH}_{3} \mathrm{OH}=1: 2\right)$ mixture was added to liver tissue and homogenized, followed by centrifugation to obtain the supernatant. Then, $100 \mu \mathrm{M}$ butylated hydroxytoluene (BHT, to prevent lipid peroxidation), $250 \mu \mathrm{l} \mathrm{CH}_{2} \mathrm{Cl}_{2}$ and $250 \mu 1$ water were sequentially added to the supernatant and vortexed for $30 \mathrm{~s}$. We transferred the lower phase, centrifuged again to take the supernatant which then evaporated to dryness under nitrogen. The residue was 
dissolved in n-hexane, $10 \mu \mathrm{l}$ internal standard (heptadecanoic acid) and $2 \mathrm{ml} 0.5 \mathrm{M} \mathrm{KOH}-$

$\mathrm{MeOH}$ were added, followed by water bath heating at $60^{\circ} \mathrm{C}$ for $20 \mathrm{~min}$. After cooling for $10 \mathrm{~min}, 3 \mathrm{ml}$ of $12.5 \% \mathrm{H}_{2} \mathrm{SO}_{4}$ in methanol solution (to methylate the sample) was added and heated again at $60^{\circ} \mathrm{C}$ in the water bath for $1 \mathrm{~h}$. After cooling the sample vial, $2 \mathrm{ml}$-hexane and $1 \mathrm{ml}$ saturated sodium chloride $(\mathrm{NaCl})$ solution were added, and let stand for $10 \mathrm{~min}$ to stratify. The supernatant (hexane fraction) was then transferred for GC-MS analysis.

GC-MS analysis was performed on Agilent 7890 A/5975 C, with specific parameter settings modified according to the previously reported procedures. The samples were separated by VF-23 ms chromatographic column (Agilent: 30m(length), $0.25 \mathrm{~mm}$ (inner diameter), $0.25 \mu \mathrm{m}$ (film thickness)). FFA composition was determined based on the retention time of validated fatty acid methyl ester standard (Supelco 37, sigma). FFA content was expressed as a percentage of peak area.

\subsection{RNA extraction, reverse transcription and real-time quantitative PCR (RT-qPCR)}

The expression of 12 genes involved in liver fatty acid metabolism (synthesis, desaturation, uptake, transport and oxidation) was determined by RT-qPCR. Total RNA was extracted from frozen liver tissue with TRIzol reagent (Invitrogen) and reverse-transcribed using PrimeScript ${ }^{\mathrm{TM}}$ RT reagent kit (Takara BIO Inc., Code No.RR047A) according to manufacturer's instructions.

RT-qPCR was performed using TB Green ${ }^{\circledR}$ Premix Ex Taq ${ }^{\text {TM }}$ II (Tli RNaseH Plus) (Takara BIO Inc., Code No. RR820A) and LightCycler ${ }^{\circledR} 96$ system. The total volume of RTqPCR reaction was $10 \mu \mathrm{L}$, and its cycling parameters were set as follows: pre-denaturation for 30 s at $95^{\circ} \mathrm{C}$, followed by 40 cycles at $95^{\circ} \mathrm{C}$ for $5 \mathrm{~s}$ and $60^{\circ} \mathrm{C}$ for $60 \mathrm{~s}$, then annealing and 
elongation. The expression level of each gene relative to GAPDH was determined by the 2-

$\Delta \Delta \mathrm{Ct}$ method, and then normalized to the corresponding control group. Gene-specific primers of hamsters for RT-qPCR are listed in table 1.

\subsection{Statistical Analysis}

Statistical comparisons of the data were performed by SPSS 18.0 software using oneway analysis of variance(ANOVA). The experimental results were presented as mean \pm standard deviation (SD). P values $<0.05$ were considered statistically significant. 


\section{Results}

\subsection{Effect of ADT on biochemical parameters and histology}

Serum ALT, AST and TBA levels were measured to assess the effect of ADT on liver

function. As shown in Figure 2A, different doses of ADT significantly reduced elevated ALT, AST, and TBA levels in hamsters fed a high-fat diet. In addition, changes in these biochemical indicators have been confirmed by pathological changes in the liver. H\&E staining results revealed that significant swelling of hepatocytes and increased lipid droplets in the liver of HFD-fed hamsters, while control hamsters displayed normal liver histology. Compared with the HFD group, liver steatosis in ADT intervention groups with different concentrations were improved to varying degrees, and liver structure tended to normal (Figure 2B).

\subsection{Quantitative changes of FFAs in hamster liver treated with ADT}

Quantitative changes of 13 FFAs detected by GC-MS in hamster liver in different experimental groups are shown in table $2(n=9)$. These data indicated that total SFA and MUFA increased significantly in the HFD group compared to the control group. However, there was no obvious change in total PUFA between the two groups, with a slight increase in n- 6 fatty acids and a decrease in n-3 fatty acids. ADT treatment significantly reduced total SFA and MUFA in the liver of hamsters fed a high-fat diet, and evidently up-regulated n-3 PUFA.

The specific changes of different FFAs between the experimental groups were further analyzed. C16:0 as well as C18:0, the two main SFAs, were increased in HFD, and this increase was subsequently inhibited by ADT treatment. C18:1n9, the main MUFA in the body, 
was significantly elevated in HFD, with concentrations nearly tripling compared to control levels, which can be reduced by ADT administration. For these three FFAs, ADT at $10 \mathrm{mg} / \mathrm{kg}$ was the most effective. In addition, C16:1 was also obviously up-regulated in HFD and was reduced in a dose-dependent manner by ADT. Meanwhile, the essential fatty acids (EFAs) including C18:2n6 and C18:3n3 were increased in HFD group and decreased in HFD + ADT group. In contrast, HFD-fed hamster livers showed lower PUFAs levels, including C20:3n6, C20:4n6 and C22:6n3, which were markedly elevated after ADT administration. Other FFAs (C14:0, C20:1, C20:2, and C24:1) had very low liver concentrations, C14:0 and C24:1 did not differ among the groups, while C20:1 and C20:2 were evidently altered.

\subsection{Effect of ADT on mRNA expression of fatty acid metabolism gene in liver}

To investigate the molecular mechanism of ADT, we further quantitatively measured mRNA expression levels of genes related to FA synthesis, desaturation, $\beta$-oxidation, uptake and transport in the liver (Figure 3). The expression of ACC1 and FAS was significantly increased in the HFD group and decreased dose-dependently in HFD + ADT groups (Figure $3 \mathrm{~A})$.

SCD1, a key rate-limiting enzyme that desaturates SFA into MUFA, was significantly elevated in the HFD group and attenuated by ADT administration (Figure 3B). The changes of SCD1 were associated with changes in C16:1/C16:0 and C18:1n9/C18:0 ratios (Table. 2). On the contrary, FADS1 and FADS2, key rate-limiting enzymes in the desaturation of PUFA, were obviously down-regulated in HFD and dose-dependently increased by ADT treatment

(Fig. 3B). Similarly, the changes in FADS1 and FADS2 were consistent with changes in C20:4n6/C18:2n6 and C22:6n3/C18:3n3 ratio(Table. 2). 
CPT $1 \alpha$ and Mitofusin1, the regulators of fatty acid $\beta$-oxidation, were concurrently decreased in HFD group, but significantly raised in the HFD + ADT group (Figure 3C).

Moreover, genes involved in liver FA uptake and transport showed that the expression of CD36 was not obviously different among the groups, while the expression of FATP2, FATP4, FATP5 and L-FABP was significantly reduced in HFD group but activated by ADT therapy (Figure 3D). 


\section{Discussion}

In this study, we investigated the effects and mechanisms of ADT on FA metabolism.

The results showed that ADT administration significantly reduced the concentrations of SFA and MUFA in HFD hamster liver, and increased the levels of n-3 PUFA. Further mechanism studies indicated that ADT treatment reduced FA synthesis, SFA desaturation, increased PUFA desaturation and promoted FAs absorption and $\beta$-oxidation, which may improving FA metabolism.

Excessive accumulation of free SFAs (Palmitic acid, PA, C16:0 and Stearic acid, SA, C18:0) in the liver can induce lipotoxicity, leading to cellular dysfunction and death(7). In our research, HFD-fed hamsters showed overexpression of ACC1 and FAS, meaning increased endogenous synthesis of PA and SA. When the increased intracellular PA level exceeds its $\beta$ oxidation in mitochondria, it is converted to harmful complex lipids such as diacylglycerin (DAG) and ceramide $(5,27)$. These harmful FA-derived intermediates and excessive PA provoke increased reactive oxygen species (ROS) production, damage the function of mitochondria-related ER membrane (MAM), and induce mitochondrial fragmentation, resulting in mitochondrial dysfunction and loss of ATP production(28-31). Recently, Rambold et al. reported that fragmented mitochondria failed to efficiently take up FA, causing a reduction in $\beta$-oxidation rates and further exacerbating the accumulation of FA in Mitofusin1 KO cells(15). Notably, this highly fused mitochondrial requirement was specific to $\beta$ oxidation, as opposed to glutamine oxidation, which was not affected by mitochondrial morphology(15). Our results indicated that ADT administration significantly inhibited ACC1 and FAS, up-regulated CPT1 $\alpha$ and Mitofusin1, thereby effectively reduced the accumulation 
of PA and SA in HFD-fed hamsters.

Oleic acid (OA, C18: 1n9), an important MUFA produced by SCD1 metabolizing SFA(32), was significantly elevated in HFD hamsters, which may be due to the body's adaptive results in the face of on-going metabolic stress. To counteract SFA-induced lipotoxicity, cells usually employ two main methods: 1 ) increasing the $\beta$-oxidation of FAs in mitochondria; and 2) inducing the storage of FAs in neutral LD (lipid droplets)(33-35). However, as mentioned above, mitochondrial $\beta$-oxidation rate decreased in HFD hamsters. The OA is mainly incorporated into relatively inert triacylglycerol (TAG) and stored in lipid droplets $(5,36)$, so metabolizing SFA to OA via SCD1 reduces the excessive formation of toxic lipid intermediates (DAG and ceramides)(37, 38). Nevertheless, this relative protection by channeling SFA into less toxic lipid pool is only temporary, as OA is less lipotoxic but more steatotic than $\mathrm{PA}(8,39)$. Continuous overproduction of OA results in liver lipid accumulation, steatosis, and low-grade chronic inflammation, thereby potentiating the metabolic syndrome $(8,39)$. In our study, ADT treatment in HFD hamsters not only effectively reduced SFAs but also significantly decreased OA.

Essential fatty acids (EFA), LA (C18:2n6) and ALA (C18:3n3) are converted to their respective PUFA metabolites by the action of FADS1 and FADS2(41). LA is converted to DGLA (C20:3n6) and AA (C20:4n6), while ALA is converted to form EPA (C20:5n3) and DHA (C22:6n3). Excessive intake of HFD reduces the levels of AA, EPA and DHA(42), enhances the production of pro-inflammatory arachidic acids and ROS, leading to proinflammatory status $(42,43)$. Our results also confirmed that HFD impaired the desaturated metabolic pathway of EFAs: the ratio of AA/LA and DHA/ALA in HFD hamsters was down- 
regulated, and the expressions of FADS1 and FADS2 were significantly decreased. In this study, the liver concentration of EPA was too low for accurate quantification. ADT administration obviously up-regulated FADS1 and FADS2, increasing the conversion of EFA to AA and DHA.

In addition to FA synthesis, $\beta$-oxidation and desaturation, the effective uptake and channeling of exogenous FA are critical to the level of FFA in hepatocytes. Evidence is emerging that specific protein transport systems are the main mediators of transmembrane FAs-trafficking into hepatocytes, including fatty acid transposase (FAT/CD 36), fatty acid transport proteins (FATPs) and liver fatty acid binding protein ( L-FABP)(44). In this study, there was no obvious difference in CD36 gene expression among the groups, while the expression of FATP2, FATP4, FATP5, and L- FABP was significantly decreased in HFD group but elevated by ADT treatment. FATP2 and FATP5 are the two main FATPs in the liver $(45,46)$. Studies have shown that sustained protein-mediated liver LCFAs uptake contributes to NFALD in cases of lipid oversupply(47). Liver specific FATP2 or FATP5 knockdown based on adeno-associated virus (AAV) significantly reduces hepatic steatosis induced by continuous high-fat feeding, thereby improving $\operatorname{NAFLD}(48,49)$. However, it is worth noting that recent clinical studies have reported that low expression of FATP5 is the most significant risk factor for liver fat $\operatorname{loss}(50)$ and is linked to the progression of $\operatorname{NAFLD}(50,51)$. Different from FATP2 and FATP5, FATP4 is localized in the liver endoplasmic reticulum and drives FAs uptake indirectly by vectorial acylation rather than acting as a transporter itself(52). Adipose or hepatocyte-specific FATP4 deficient mice under high fat/sugar diets exhibited a higher degree of hepatic steatosis $(53,54)$. 
Whether increased FATP-mediated LCFAs uptake is salutary or detrimental may depend on duration, tissue type, and subsequent fate of FAs. Upon entering hepatocytes, LCFAs and LCFA-CoA are bound/isolated by L-FABP to minimize the toxic effects of excess free FAs(55). L-FABP is a vital endogenous cytoprotectant that transports bound LCFAs for rapid removal in various cellular compartments(56-59), including endoplasmic reticulum, lipid vesicles, peroxisomes, mitochondria, and nucleus. More notably, L-FABP is not only the intracellular counterpart of albumin but also has strong antioxidant properties(56, 60, 61). Studies have shown that the loss of L-FABP may enhance the production of lipotoxic inflammatory mediators, impair the oxidative pathway of FAs, and render hepatocytes more vulnerable to the harmful effects of LCFAs, thus promoting the development of NAFLD(62, 63). Consistent with these studies, the expression of L-FABP and CPT1 $\alpha$ was significantly reduced in HFD-fed hamsters, which suggested that delivery of FAs to mitochondria was inhibited, thereby impairing oxidation and leading to overaccumulation of FAs. ADT treatment significantly up-regulated the expression of L-FABP and CPT1 $1 \alpha$, therefore improving the $\beta$-oxidation of FAs in the liver.

In conclusion, this study demonstrates that ADT is effective in improving HFD induced fatty acid metabolism disorders. Specifically, ADT may exert protective effects by: (i) inhibiting overexpression of ACC1, FAS, and SCD1, thereby reducing endogenous synthesis of toxic palmic acid (C16:0) and oleic acid (C18:1); (ii) up-regulating FATPs, L-FABP, CPT $1 \alpha$ and Mitofusin1, thus enhancing the $\beta$-oxidation of liver fatty acids; (iii) activating FADS1 and FADS2 to improve the desaturated metabolic pathway of EFAs (Figure 4). These results may provide important new insights into the role of ADT in hepatic lipid metabolism, 
but further researchs are still needed to evaluate its potential application in clinical treatment of hyperlipidemia and NAFLD.

\section{Conflicts Of Interest}

The authors declare that they have no competing interests. 


\section{References:}

1. Masoodi M, Kuda O, Rossmeisl M, Flachs P, Kopecky J. 2015. Lipid signaling in adipose tissue: Connecting inflammation \&amp; metabolism. Biochim Biophys Acta. 1851:503-518.

2. Wang Y, Qian Y, Fang Q, Zhong P, Li W, Wang L, Fu W, Zhang Y, Xu Z, Li X, Liang

G. 2017. Saturated palmitic acid induces myocardial inflammatory injuries through direct binding to TLR4 accessory protein MD2. Nat Commun. 8:13997.

3. Lu H, Hao L, Li S, Lin S, Lv L, Chen Y, Cui H, Zi T, Chu X, Na L, Sun C. 2016.

Elevated circulating stearic acid leads to a major lipotoxic effect on mouse pancreatic beta cells in hyperlipidaemia via a miR-34a-5p-mediated PERK/p53-dependent pathway.

Diabetologia. 59:1247-1257.

4. Cansancao K, Silva ML, Carvalho LN, Davalos A, Tavares DCM, Arantes FPW. 2018. Advanced Liver Fibrosis Is Independently Associated with Palmitic Acid and Insulin Levels in Patients with Non-Alcoholic Fatty Liver Disease. Nutrients. 10:

5. Palomer X, Pizarro-Delgado J, Barroso E, Vazquez-Carrera M. 2018. Palmitic and Oleic Acid: The Yin and Yang of Fatty Acids in Type 2 Diabetes Mellitus. Trends Endocrinol Metab. 29:178-190.

6. Steffen BT, Duprez D, Szklo M, Guan W, Tsai MY. 2018. Circulating oleic acid levels are related to greater risks of cardiovascular events and all-cause mortality: The Multi-Ethnic Study of Atherosclerosis. J. Clin Lipidol. 12:1404-1412.

7. Leamy AK, Egnatchik RA, Young JD. 2013. Molecular mechanisms and the role of saturated fatty acids in the progression of non-alcoholic fatty liver disease. Prog. Lipid Res. $52: 165-174$ 
8. Ricchi M, Odoardi MR, Carulli L, Anzivino C, Ballestri S, Pinetti A, Fantoni LI, Marra F, Bertolotti M, Banni S, Lonardo A, Carulli N, Loria P. 2009. Differential effect of oleic and palmitic acid on lipid accumulation and apoptosis in cultured hepatocytes. $J$ Gastroenterol Hepatol. 24:830-840.

9. Cheon HG, Cho YS. 2014. Protection of palmitic acid-mediated lipotoxicity by arachidonic acid via channeling of palmitic acid into triglycerides in C2C12. J. Biomed. Sci. $21: 13$.

10. Chaurasia B, Summers SA. 2015. Ceramides - Lipotoxic Inducers of Metabolic Disorders. Trends Endocrinol Metab. 26:538-550.

11. Salvado L, Palomer X, Barroso E, Vazquez-Carrera M. 2015. Targeting endoplasmic reticulum stress in insulin resistance. Trends Endocrinol Metab. 26:438-448.

12. Win S, Than TA, Le BH, Garcia-Ruiz C, Fernandez-Checa JC, Kaplowitz N. 2015. Sab (Sh3bp5) dependence of JNK mediated inhibition of mitochondrial respiration in palmitic acid induced hepatocyte lipotoxicity. J. Hepatol. 62:1367-1374.

13. Sharma G, Parihar A, Parihar P, Parihar MS. 2019. Downregulation of sirtuin 3 by palmitic acid increases the oxidative stress, impairment of mitochondrial function, and apoptosis in liver cells. J Biochem Mol Toxicol. 33:e22337.

14. Velloso LA, Folli F, Saad MJ. 2015. TLR4 at the Crossroads of Nutrients, Gut Microbiota, and Metabolic Inflammation. Endocr. Rev. 36:245-271.

15. Rambold AS, Cohen S, Lippincott-Schwartz J. 2015. Fatty acid trafficking in starved cells: regulation by lipid droplet lipolysis, autophagy, and mitochondrial fusion dynamics. Dev. Cell. 32:678-692. 
16. Wrighton KH. 2015. Lipid metabolism: fatty acids on the move. Nat Rev Mol Cell Biol. $16: 204$.

17. Li M, Xu C, Shi J, Ding J, Wan X, Chen D, Gao J, Li C, Zhang J, Lin Y, Tu Z, Kong X, Li Y, Yu C. 2017. Fatty acids promote fatty liver disease via the dysregulation of 3mercaptopyruvate sulfurtransferase/hydrogen sulfide pathway. Gut. 67:2169-2180.

18. Xie L, Gu Y, Wen M, Zhao S, Wang W, Ma Y, Meng G, Han Y, Wang Y, Liu G, Moore PK, Wang X, Wang H, Zhang Z, Yu Y, Ferro A, Huang Z, Ji Y. 2016. Hydrogen Sulfide Induces Keap1 S-sulfhydration and Suppresses Diabetes-Accelerated Atherosclerosis via Nrf2 Activation. Diabetes. 65:3171-3184.

19. Liu Z, Han Y, Li L, Lu H, Meng G, Li X, Shirhan M, Peh MT, Xie L, Zhou S, Wang X, Chen Q, Dai W, Tan CH, Pan S, Moore PK, Ji Y. 2013. The hydrogen sulfide donor, GYY4137, exhibits anti-atherosclerotic activity in high fat fed apolipoprotein E(-/-) mice. $\mathrm{Br}$ J Pharmacol. 169:1795-1809.

20. Xue R, Hao DD, Sun JP, Li WW, Zhao MM, Li XH, Chen Y, Zhu JH, Ding YJ, Liu J, Zhu YC. 2013. Hydrogen sulfide treatment promotes glucose uptake by increasing insulin receptor sensitivity and ameliorates kidney lesions in type 2 diabetes. Antioxid Redox Signal. 19:5-23.

21. Sun L, Zhang S, Yu C, Pan Z, Liu Y, Zhao J, Wang X, Yun F, Zhao H, Yan S, Yuan Y, Wang D, Ding X, Liu G, Li W, Zhao X, Liu Z, Li Y. 2015. Hydrogen sulfide reduces serum triglyceride by activating liver autophagy via the AMPK-mTOR pathway. Am J Physiol Endocrinol Metab. 309:E925-E935.

22. Li L, Whiteman M, Guan YY, Neo KL, Cheng Y, Lee SW, Zhao Y, Baskar R, Tan CH, 
Moore PK. 2008. Characterization of a novel, water-soluble hydrogen sulfide-releasing molecule (GYY4137): new insights into the biology of hydrogen sulfide. Circulation. 117:2351-2360.

23. Szabo C, Papapetropoulos A. 2017. International Union of Basic and Clinical Pharmacology. CII: Pharmacological Modulation of H2S Levels: H2S Donors and H2S Biosynthesis Inhibitors. Pharmacol. Rev. 69:497-564.

24. Wang Y, Jia J, Ao G, Hu L, Liu H, Xiao Y, Du H, Alkayed NJ, Liu CF, Cheng J. 2014. Hydrogen sulfide protects blood-brain barrier integrity following cerebral ischemia. $J$. Neurochem. 129:827-838.

25. Huang WC, Chen YM, Kan NW, Ho CS, Wei L, Chan CH, Huang HY, Huang CC. 2015. Hypolipidemic effects and safety of Lactobacillus reuteri 263 in a hamster model of hyperlipidemia. Nutrients. 7:3767-3782.

26. Tang M, Liu Y, Wang L, Li H, Cai H, Zhang M, Dang R, Xue Y, Wu Y. 2018. An Omega-3 fatty acid-deficient diet during gestation induces depressive-like behavior in rats: the role of the hypothalamo-pituitary-adrenal (HPA) system. Food Funct. 9:3481-3488. 27. Chavez JA, Summers SA. 2003. Characterizing the effects of saturated fatty acids on insulin signaling and ceramide and diacylglycerol accumulation in 3T3-L1 adipocytes and C2C12 myotubes. Arch. Biochem. Biophys. 419:101-109.

28. Li Z, Berk M, McIntyre TM, Gores GJ, Feldstein AE. 2008. The lysosomal-mitochondrial axis in free fatty acid-induced hepatic lipotoxicity. Hepatology. 47:1495-1503.

29. Korbecki J, Bajdak-Rusinek K. 2019. The effect of palmitic acid on inflammatory response in macrophages: an overview of molecular mechanisms. Inflamm. Res. 68:915-932. 
30. Shinjo S, Jiang S, Nameta M, Suzuki T, Kanai M, Nomura Y, Goda N. 2017. Disruption of the mitochondria-associated ER membrane (MAM) plays a central role in palmitic acidinduced insulin resistance. Exp. Cell Res. 359:86-93.

31. Ohtsu A, Tanaka H, Seno K, Iwata H, Kuwayama T, Shirasuna K. 2017. Palmitic acid stimulates interleukin-8 via the TLR4/NF-kappaB/ROS pathway and induces mitochondrial dysfunction in bovine oviduct epithelial cells. Am. J. Reprod. Immunol. 77:

32. ALJohani AM, Syed DN, Ntambi JM. 2017. Insights into Stearoyl-CoA Desaturase-1 Regulation of Systemic Metabolism. Trends Endocrinol Metab. 28:831-842.

33. Sinha RA, Singh BK, Zhou J, Xie S, Farah BL, Lesmana R, Ohba K, Tripathi M, Ghosh S, Hollenberg AN, Yen PM. 2017. Loss of ULK1 increases RPS6KB1-NCOR1 repression of NR1H/LXR-mediated Scd1 transcription and augments lipotoxicity in hepatic cells. Autophagy. 13:169-186.

34. Asai T, Okumura K, Takahashi R, Matsui H, Numaguchi Y, Murakami H, Murakami R, Murohara T. 2006. Combined therapy with PPARalpha agonist and L-carnitine rescues lipotoxic cardiomyopathy due to systemic carnitine deficiency. Cardiovasc. Res. 70:566-577.

35. Listenberger LL, Han X, Lewis SE, Cases S, Farese RJ, Ory DS, Schaffer JE. 2003.

Triglyceride accumulation protects against fatty acid-induced lipotoxicity. Proc Natl Acad Sci US A. 100:3077-3082.

36. Man WC, Miyazaki M, Chu K, Ntambi J. 2006. Colocalization of SCD1 and DGAT2: implying preference for endogenous monounsaturated fatty acids in triglyceride synthesis. $J$. Lipid Res. 47:1928-1939.

37. Piccinin E, Cariello M, De Santis S, Ducheix S, Sabba C, Ntambi JM, Moschetta A. 2019. 
Role of Oleic Acid in the Gut-Liver Axis: From Diet to the Regulation of Its Synthesis via Stearoyl-CoA Desaturase 1 (SCD1). Nutrients. 11:E2283.

38. Peng G, Li L, Liu Y, Pu J, Zhang S, Yu J, Zhao J, Liu P. 2011. Oleate blocks palmitateinduced abnormal lipid distribution, endoplasmic reticulum expansion and stress, and insulin resistance in skeletal muscle. Endocrinology. 152:2206-2218.

39. Hoang NA, Richter F, Schubert M, Lorkowski S, Klotz LO, Steinbrenner H. 2018.

Differential capability of metabolic substrates to promote hepatocellular lipid accumulation.

Eur. J. Nutr. 58:3023-3034.

40. Singh V, Chassaing B, Zhang L, San YB, Xiao X, Kumar M, Baker MT, Cai J, Walker R, Borkowski K, Harvatine KJ, Singh N, Shearer GC, Ntambi JM, Joe B, Patterson AD, Gewirtz AT, Vijay-Kumar M. 2015. Microbiota-Dependent Hepatic Lipogenesis Mediated by Stearoyl CoA Desaturase 1 (SCD1) Promotes Metabolic Syndrome in TLR5-Deficient Mice. Cell Metab. 22:983-996.

41. Koletzko B, Reischl E, Tanjung C, Gonzalez-Casanova I, Ramakrishnan U, Meldrum S, Simmer K, Heinrich J, Demmelmair H. 2019. FADS1 and FADS2 Polymorphisms Modulate Fatty Acid Metabolism and Dietary Impact on Health. Annu Rev Nutr. 39:21-44.

42. Das UN. 2018. Ageing: Is there a role for arachidonic acid and other bioactive lipids? A review. J. Adv Res. 11:67-79.

43. Das UN. 2018. Arachidonic acid in health and disease with focus on hypertension and diabetes mellitus: A review. J. Adv Res. 11:43-55.

44. Doege H, Stahl A. 2006. Protein-mediated fatty acid uptake: novel insights from in vivo models. Physiology (Bethesda). 21:259-268. 
45. Hirsch D, Stahl A, Lodish HF. 1998. A family of fatty acid transporters conserved from mycobacterium to man. Proc Natl Acad Sci U S A. 95:8625-8629.

46. Doege H, Baillie RA, Ortegon AM, Tsang B, Wu Q, Punreddy S, Hirsch D, Watson N, Gimeno RE, Stahl A. 2006. Targeted deletion of FATP5 reveals multiple functions in liver metabolism: alterations in hepatic lipid homeostasis. Gastroenterology. 130:1245-1258. 47. Mun J, Kim S, Yoon HG, You Y, Kim OK, Choi KC, Lee YH, Lee J, Park J, Jun W. 2019. Water Extract of Curcuma longa L. Ameliorates Non-Alcoholic Fatty Liver Disease. Nutrients. 11:E2536.

48. Falcon A, Doege H, Fluitt A, Tsang B, Watson N, Kay MA, Stahl A. 2010. FATP2 is a hepatic fatty acid transporter and peroxisomal very long-chain acyl-CoA synthetase. Am $J$ Physiol Endocrinol Metab. 299:E384-E393.

49. Doege H, Grimm D, Falcon A, Tsang B, Storm TA, Xu H, Ortegon AM, Kazantzis M, Kay MA, Stahl A. 2008. Silencing of hepatic fatty acid transporter protein 5 in vivo reverses diet-induced non-alcoholic fatty liver disease and improves hyperglycemia. J. Biol. Chem. 283:22186-22192.

50. Enooku K, Tsutsumi T, Kondo M, Fujiwara N, Sasako T, Shibahara J, Kado A, Okushin K, Fujinaga H, Nakagomi R, Minami T, Sato M, Uchino K, Nakagawa H, Kondo Y, Asaoka Y, Tateishi R, Ueki K, Ikeda H, Yoshida H, Moriya K, Yotsuyanagi H, Kadowaki T, Koike K. 2019. Hepatic FATP5 expression is associated with histological progression and loss of hepatic fat in NAFLD patients. J. Gastroenterol. 55:227-243.

51. Mitsuyoshi H, Yasui K, Harano Y, Endo M, Tsuji K, Minami M, Itoh Y, Okanoue T, Yoshikawa T. 2009. Analysis of hepatic genes involved in the metabolism of fatty acids and 
iron in nonalcoholic fatty liver disease. Hepatol. Res. 39:366-373.

52. Milger K, Herrmann T, Becker C, Gotthardt D, Zickwolf J, Ehehalt R, Watkins PA, Stremmel W, Fullekrug J. 2006. Cellular uptake of fatty acids driven by the ER-localized acyl-CoA synthetase FATP4. J. Cell Sci. 119:4678-4688.

53. Lenz LS, Marx J, Chamulitrat W, Kaiser I, Grone HJ, Liebisch G, Schmitz G, Elsing C, Straub BK, Fullekrug J, Stremmel W, Herrmann T. 2011. Adipocyte-specific inactivation of Acyl-CoA synthetase fatty acid transport protein 4 (Fatp4) in mice causes adipose hypertrophy and alterations in metabolism of complex lipids under high fat diet. J. Biol. Chem. 286:35578-35587.

54. Doring S, Seessle J, Gan-Schreier H, Javaheri B, Jiao L, Cheng Y, Tuma-Kellner S, Liebisch G, Herrmann T, Stremmel W, Chamulitrat W. 2019. Elevation of blood lipids in hepatocyte-specific fatty acid transport 4-deficient mice fed with high glucose diets. Mol. Genet. Metab. 126:30-38.

55. Atshaves BP, Martin GG, Hostetler HA, McIntosh AL, Kier AB, Schroeder F. 2010. Liver fatty acid-binding protein and obesity. J. Nutr. Biochem. 21:1015-1032.

56. Wang G, Bonkovsky HL, de Lemos A, Burczynski FJ. 2015. Recent insights into the biological functions of liver fatty acid binding protein 1. J. Lipid Res. 56:2238-2247.

57. Wolfrum C, Borrmann CM, Borchers T, Spener F. 2001. Fatty acids and hypolipidemic drugs regulate peroxisome proliferator-activated receptors alpha - and gamma-mediated gene expression via liver fatty acid binding protein: a signaling path to the nucleus. Proc Natl Acad Sci U S A. 98:2323-2328.

58. Bhuiyan AK, Pande SV. 1994. Carnitine palmitoyltransferase activities: effects of serum 
albumin, acyl-CoA binding protein and fatty acid binding protein. Mol. Cell. Biochem. 139:109-116.

59. Thumser AE, Storch J. 2007. Characterization of a BODIPY-labeled fluorescent fatty acid analogue. Binding to fatty acid-binding proteins, intracellular localization, and metabolism. Mol. Cell. Biochem. 299:67-73.

60. Yan J, Gong Y, She YM, Wang G, Roberts MS, Burczynski FJ. 2009. Molecular mechanism of recombinant liver fatty acid binding protein's antioxidant activity. J. Lipid Res. $50: 2445-2454$.

61. Smathers RL, Galligan JJ, Shearn CT, Fritz KS, Mercer K, Ronis M, Orlicky DJ, Davidson NO, Petersen DR. 2013. Susceptibility of L-FABP-/- mice to oxidative stress in early-stage alcoholic liver. J. Lipid Res. 54:1335-1345.

62. Newberry EP, Xie Y, Lodeiro C, Solis R, Moritz W, Kennedy S, Barron L, Onufer E, Alpini G, Zhou T, Blaner WS, Chen A, Davidson NO. 2019. Hepatocyte and stellate cell deletion of liver fatty acid binding protein reveals distinct roles in fibrogenic injury. Faseb $J$. $33: 4610-4625$.

63. Charlton M, Viker K, Krishnan A, Sanderson S, Veldt B, Kaalsbeek AJ, Kendrick M, Thompson G, Que F, Swain J, Sarr M. 2009. Differential expression of lumican and fatty acid binding protein-1: new insights into the histologic spectrum of nonalcoholic fatty liver disease. Hepatology. 49:1375-1384. 
Table 1

Table 1. Hamster primer sequences used in this study.

\begin{tabular}{lll}
\hline Gene & Forward primer & Reverse primer \\
\hline ACC1 & 5'-TCAAGTCCTTCCTGCTCACACA-3' & 5'-TCCACCATCACTCAGCCGAT-3' \\
FAS & 5'-TTAGCTCTAGTCCCACCCGGAA-3' & 5'-CACTAGACTCCAGCAGATTAACCC-3' \\
SCD1 & 5'-GGTACTACAAGCCCGCCAT-3' & 5'-AGCACCAAAGTGTATCGCAAG-3' \\
FADS1 & 5'-ACCTCTTTTAATCAGTCCCCAA-3' & 5'-GCTATACAATGCTGGAACACA-3' \\
FADS2 & 5'-GCACCTCAACTTCCAGATCGAG-3' & 5'-CAGGGAACTCACAATGTCCAGCAG-3' \\
CPT1 $\alpha$ & 5'-GGTTTGACAAGTCCCTCACGTT-3' & 5'-TCTCCTTTACAATGCCCGTCCT-3' \\
Mitofusin1 & 5'-TCTTAACAACAAAGGCTGCTCT-3' & 5'-TCATTACCACAGTCTCGGCAAG-3' \\
CD36 & 5'-CAAATGCAAAGAAGGAAAGCCTGT-3' & 5'-GGCTCCACATCCAAGTATGTCC-3' \\
FATP2 & 5'-TTAAACACCGCAAAGTGACCCT-3' & 5'-TCACCGGGATACTCAGAGCTT-3' \\
FATP4 & 5'-GGCAATCAATCTGGACCGACT-3' & 5'-ACACAAAAGACAGGATTCGGCTA-3' \\
FATP5 & 5'-CACACCTCATTTCATCCGCATC-3' & 5'-GTCATAGCTTCCACGTTCCCTC-3' \\
L-FABP & 5'-ATCAGAAATCGAGCATAACGGGAA-3' & 5'-CATCTTAACCACAGCCTTGACC-3' \\
GAPDH & 5'-TTGCTGCCATCAATGACCCCTT-3' & 5'-TTCTCAGCCTTGACTGTGCCTT-3' \\
\hline
\end{tabular}




\section{Table 2}

Table 2. Quantitative changes of liver FFAs in hamsters treated with ADT $(n=9)$

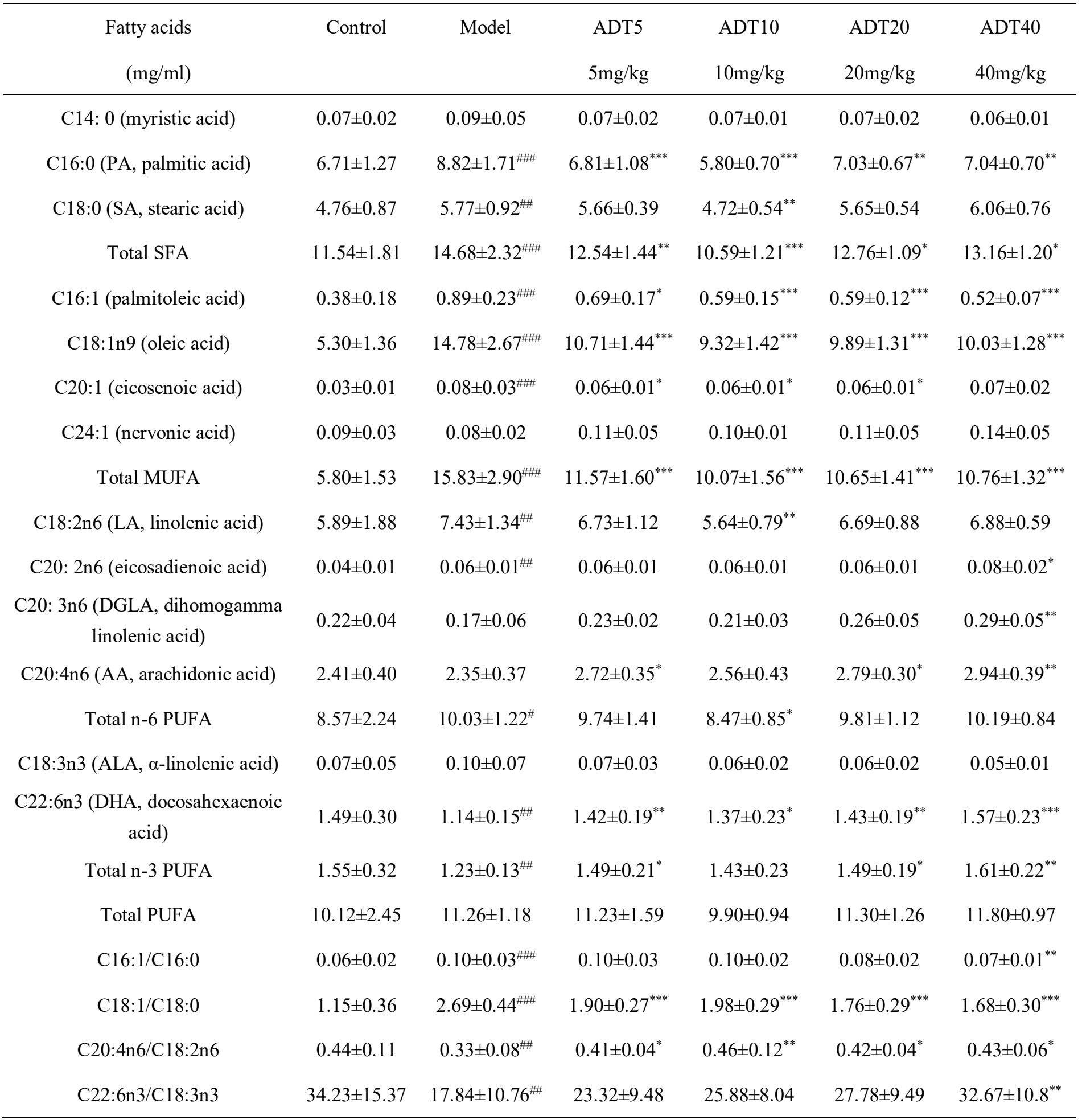

Results are presented as mean $\pm S D$. SFA correspond to 14:0, 16:0 and 18:0. MUFA

correspond to $16: 1,18: 1 \mathrm{n} 9,20: 1$ and 24:1. n-6 PUFA include 18:2n6, 20:2n6, 20:3n6 and 
20:4n6; n-3 PUFA include 18:3n3 and 22:6n3; PUFA correspond to n-6 and n-3 PUFA.

${ }^{\#} p<0.05,{ }^{\# \#} p<0.01$, and ${ }^{\# \#} p<0.001$ versus control. ${ }^{*} p<0.05, \stackrel{* *}{p}<0.01$, and ${ }^{* * *} p<0.001$

versus HFD. 


\section{Figure 1}

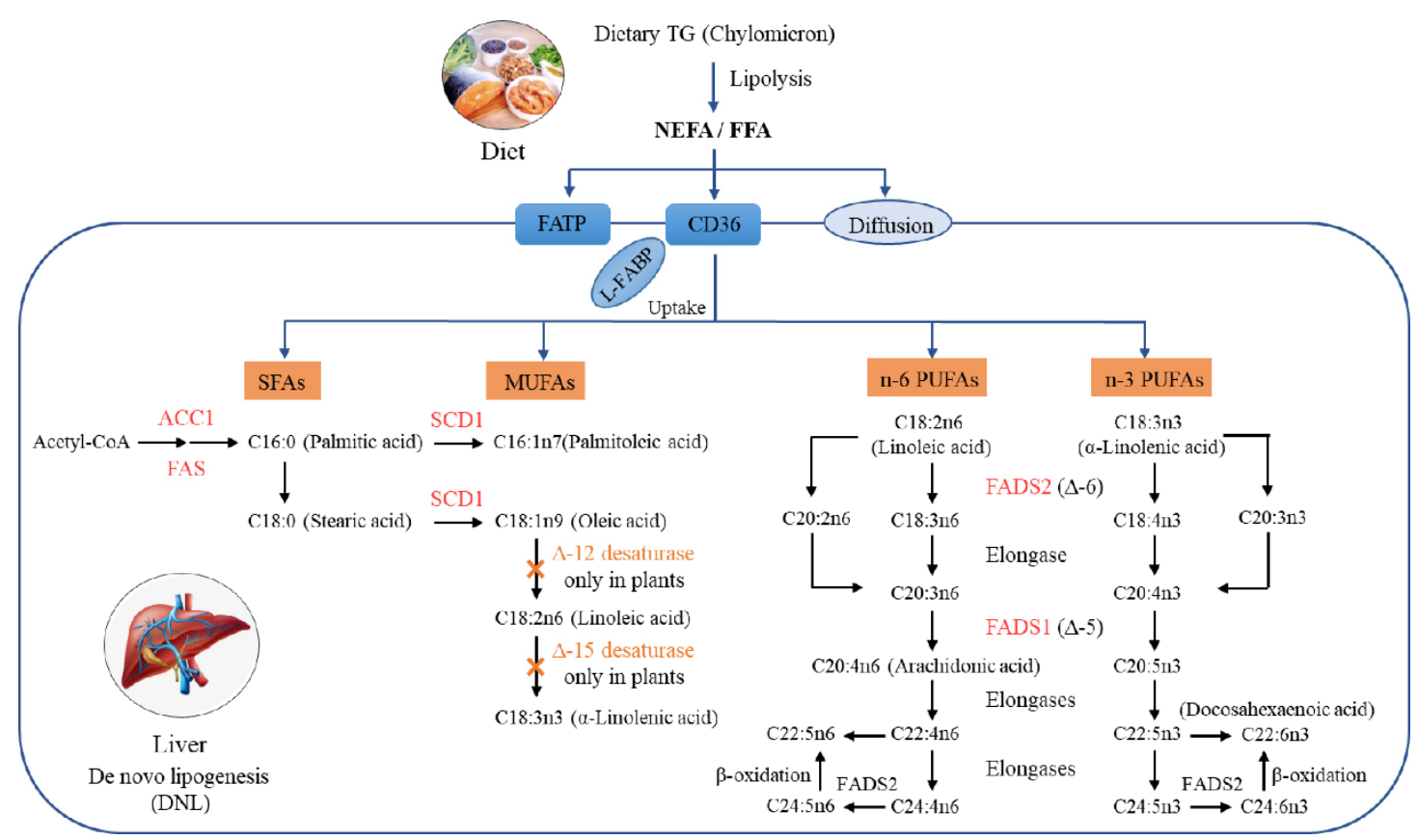

Figure 1. Metabolism of liver FAs. The metabolism of FAs is a complex process, involving multiple enzymes of synthesis, desaturation, elongation, and oxidation. Shown here are pathways for liver SFAs, MUFAs, n-6 and n-3 PUFAs synthesis and metabolism. 


\section{Figure 2}
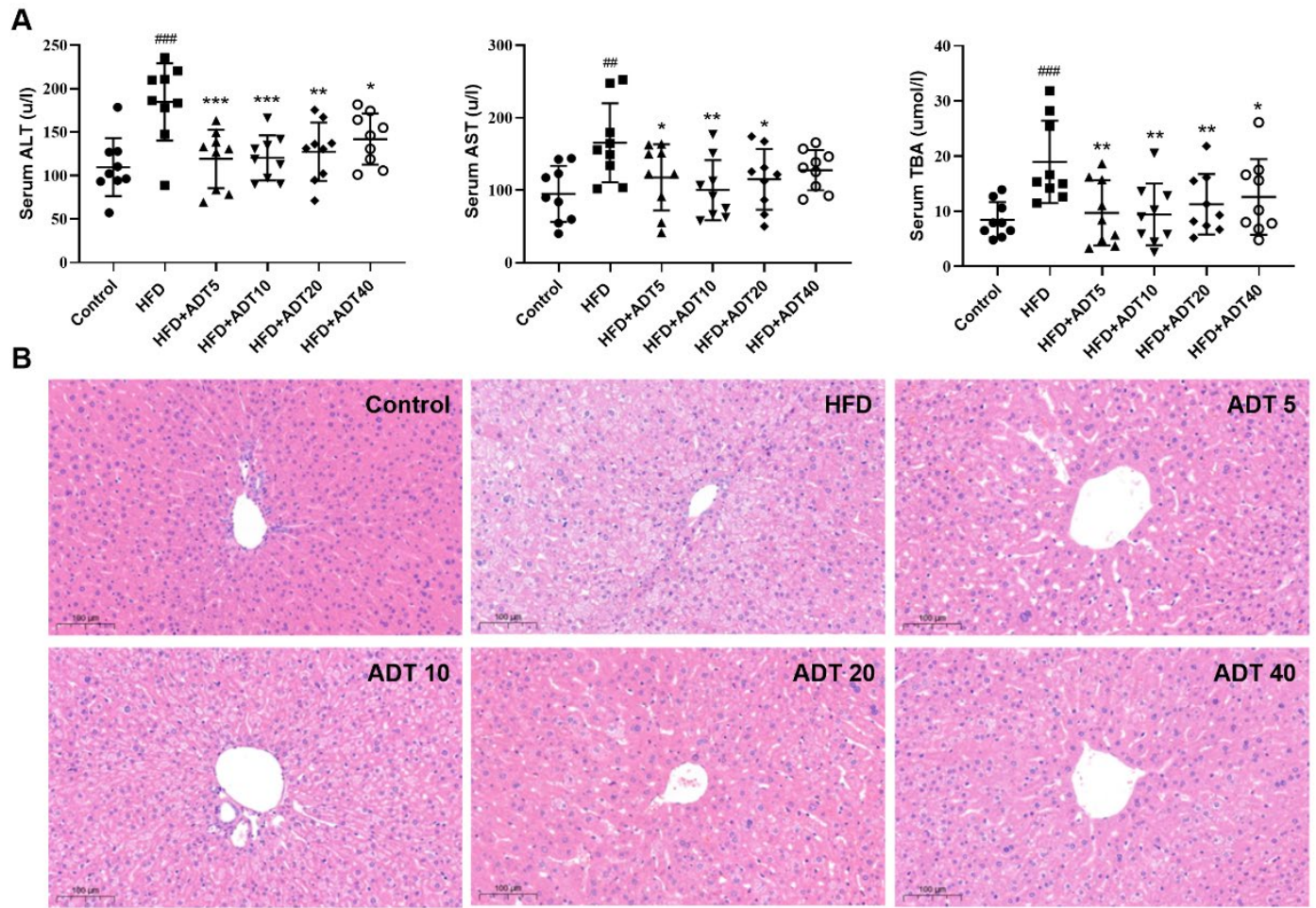

Figure 2. Effect of ADT on liver function of HFD-fed hamsters. (A): Serum levels of ALT,

AST, and TBA in each group of hamsters $(\mathrm{n}=9)$. Data are presented as mean $\pm S D$. ALT $=$

alanine aminotransferase; $\mathrm{AST}=$ aspartate aminotransferase; $\mathrm{TBA}=$ total biliary acid.

${ }^{\#} p<0.05,{ }^{\#} p<0.01$, and ${ }^{\# \#} p<0.001$ versus control. ${ }^{*} p<0.05,{ }^{* *} p<0.01$, and ${ }^{* * *} p<0.001$

versus HFD. (B): Histopathological examination of hamster liver sections by HE staining

(magnification, $\times 100)$. 
Figure 3
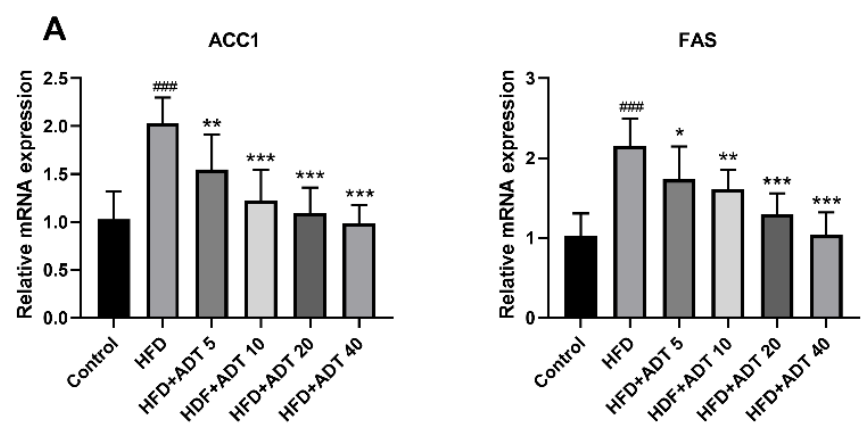

B

SCD1

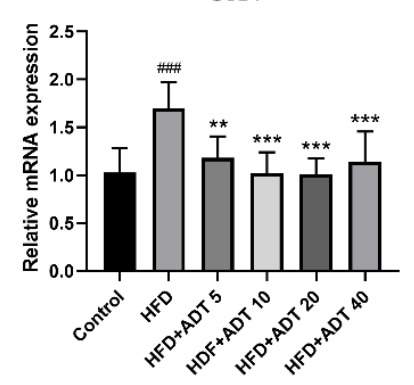

FADS1
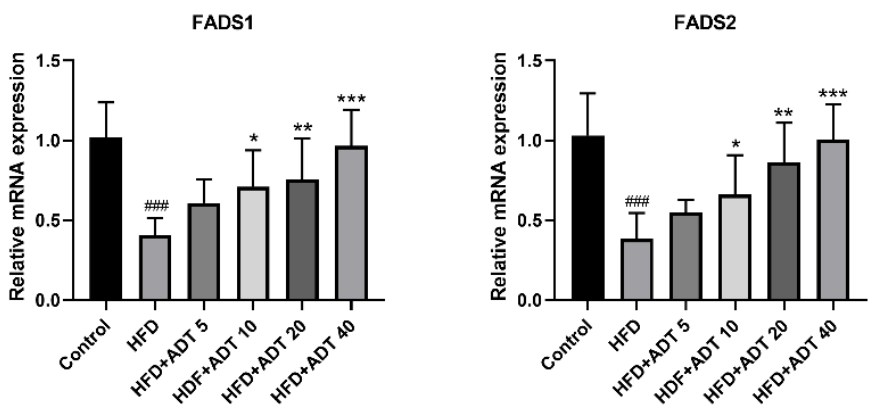

C

CPT1a
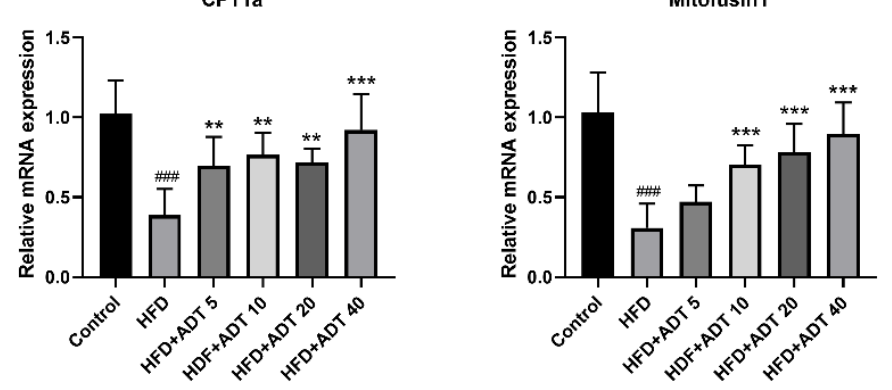

。

CD36
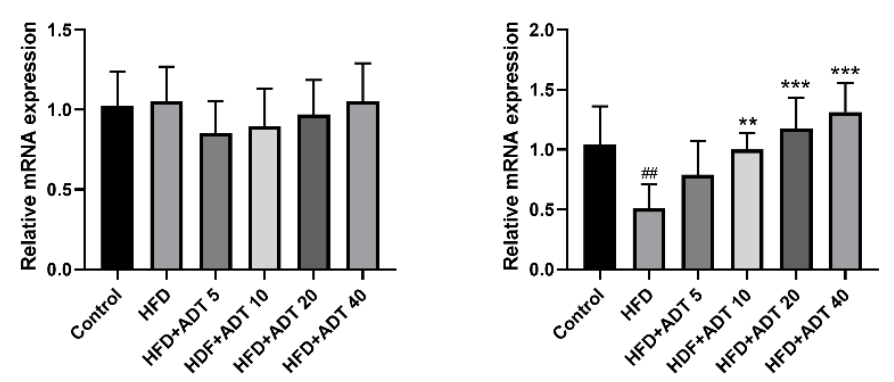

FATP2
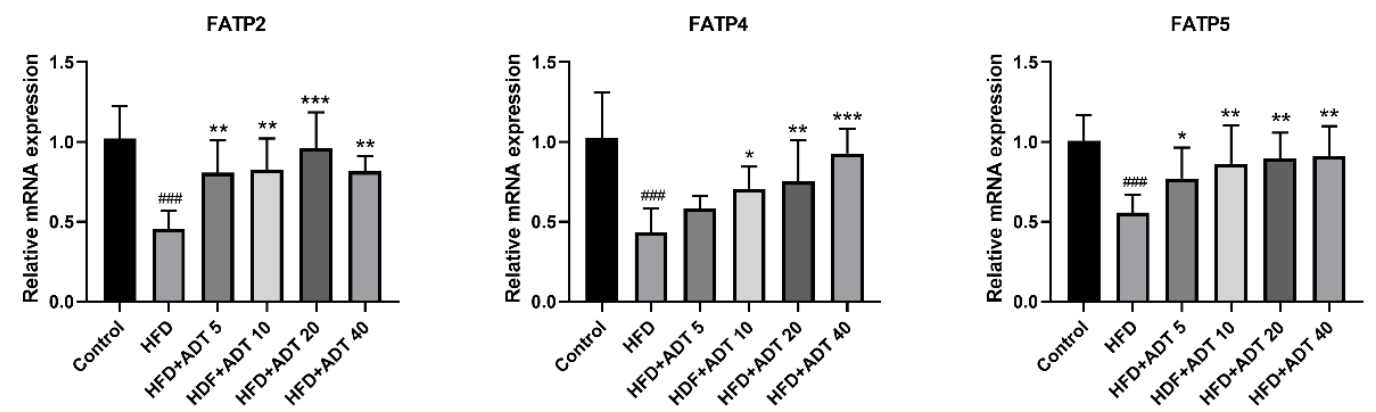

Figure 3. mRNA levels of genes related to FAs metabolism in hamster liver ( $n=6)$. (A). FAs 
synthesis: $\mathrm{ACCl}$ and FAS. (B). FAs desaturation: SCD1 converts SFA into MUFA, with the main product is C18:1n9; FADS1 and FADS2 metabolize EFAs(LA and ALA) into more unsaturated long-chain PUFAs. (C). FAs $\beta$-oxidation: CPT1 $\alpha$ catalyzes FAs into mitochondria; Mitofusin1 regulates the fusion state of mitochondria. (D). FAs uptake and transport: CD36, FATP2, FATP4, FATP5 and L-FABP. ${ }^{\#} p<0.05,{ }^{\# \#} p<0.01$, and ${ }^{\# \# \#} p<0.001$ versus control. ${ }^{*} p<0.05,{ }^{* *} p<0.01$, and ${ }^{* * *} p<0.001$ versus HFD. 


\section{Figure 4}
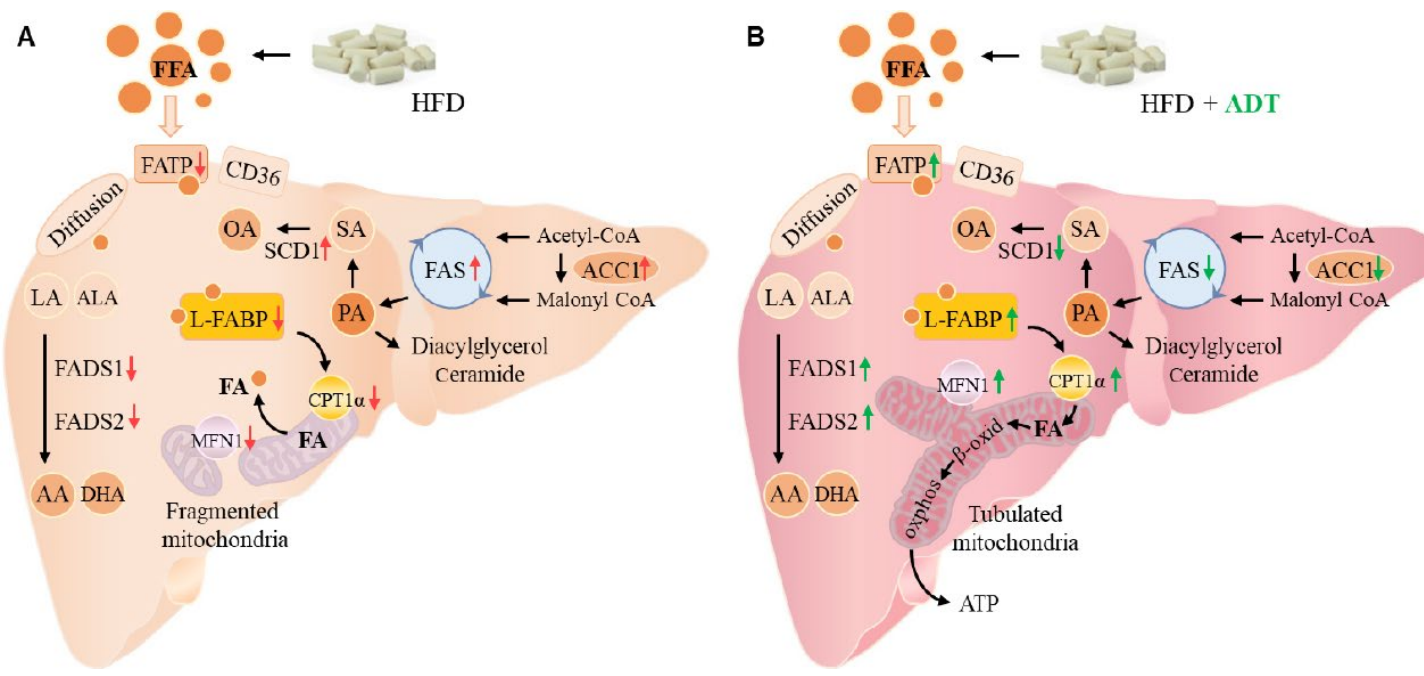

Figure 4. The mechanistic flowchart of ADT on liver FAs metabolism in HFD hamsters.

Compared with the HFD group, ADT administration significantly inhibited the

overexpression of ACC1, FAS and SCD1, activated FADS1 and FADS2, thereby reducing

endogenous synthesis of PA, SA and OA, and improved the desaturated metabolic pathway of

EFAs. In addition, ADT upregulated the levels of FATPs, L-FABP, and CPT1 $\alpha$, thus

increasing the uptake, binding/isolation, transport, and $\beta$-oxidation of FAs. More importantly,

ADT obviously increased the expression of Mitofusin1 to promote mitochondrial fusion state

and maximal $\beta$-oxidation. $\mathrm{PA}=$ Palmitic acid; $\mathrm{SA}=$ Stearic acid; $\mathrm{OA}=$ Oleic acid; $\mathrm{EFAs}=$

Essential fatty acids, including LA and ALA; MFN1= Mitofusin1. 